\title{
Hidrops fetalis no immune: A propósito de un caso
}

\author{
JOEL CÁRDENAS ${ }^{1,4}$, ERASMO HUERTAS ${ }^{1,2,4}$, ISMENIA ORÉ $^{1,3}$, SEMIRAMIS CARBAJAL $^{1}$ \\ ${ }^{1}$ Instituto Especializado Materno Perinatal. ${ }^{2}$ Unidad de Medicina Materno Fetal. ${ }^{3}$ Laboratorio de Citogenética, \\ ${ }^{4}$ Facultad de Medicina, Universidad Nacional Mayor de San Marcos
}

\begin{abstract}
Resumen
Presentamos un caso de hidrops fetalis no inmune por trisomía del par 21, diagnosticado a las 31 semanas de gestación. Mediante cordocentesis y estudio cromosómico de linfocitos se llegó al diagnóstico etiológico de síndrome Down. La paciente reingresó a los 6 días del procedimiento con diagnóstico de óbito fetal, produciéndose el parto vaginal electivo al siguiente día. La importancia de este trabajo radica en destacar la factibilidad del diagnóstico citogenético antenatal en nuestro medio mediante técnicas invasivas.
\end{abstract}

Palabras clave: Hydrops fetalis; edema; síndrome de Down; enfermedades fetales; perinatología.

\begin{abstract}
Non-immune hidrops fetalis: Apropos of a case
\section{Abstract}

We present a case of non-immune hydrops fetalis diagnosed by antenatal ultrasound at 31 weeks of gestation . Fetal karyotyping of blood sample obtained by cordocentesis was abnormal. The fetus died 6 days later. Postmortem examination was compatible with Down syndrome. We report the possibility of antenatal hydrops fetalis diagnosis by invasive techniques.
\end{abstract}

Key words: Hydrops fetalis; edema; Down syndrome; fetal diseases; perinatology.

\section{INTRODUCCIÓN}

El hidrops fetalis es un grave proceso, de elevada mortalidad perinatal, de etiología multifactorial y caracterizado por anasarca generalizada, con o sin acumulación de líquido en las cavidades serosas del organismo fetal, con algún grado de edema cutáneo y, frecuentemente, con placenta edematosa ${ }^{1}$ ). Hipócrates describió el primer caso. Pero, fue Ballantyne, en 1892, quien estableció los criterios clinicopatológicos para el diagnóstico de hidropesía fetal. La incidencia varía de 1 en 1600 a 1 en $7000\left(^{2}\right)$. Presentamos el primer

Correspondencia:

Dr. Segundo Joel Cárdenas Goicoechea

Av. Faucett Cdra. 32, Mz K-19. Urb. Aeropuerto

Callao 1, Perú.

E-mail: joelcardenas@yahoo.com caso de diagnóstico citogenético antenatal de síndrome de Down, en el Instituto Materno Perinatal (IMP).

\section{REPORTE DEL CASO}

Segundigesta de 25 años de edad, acude por primera vez al IMP referida de un centro de salud a las 31 semanas de gestación por la presencia de polihidramnios e hidrops fetalis en una ecografía de control. La paciente no tuvo hijo con malformaciones en la gestación previa. Es hospitalizada para estudio. Al examen físico se encontró una presión arterial de 110/60, frecuencia de pulso 96 , frecuencia respiratoria 18 y temperatura $37^{\circ} \mathrm{C}$; altura uterina $30 \mathrm{~cm}$, longitudinal cefálico derecho, latidos fetales 152 por minuto, con cierta disnea. Se realizó una ecografía en la Unidad de Medicina Fetal 
(UMF), encontrándose: gestación única activa de 31 semanas, polihidramnios (índice de líquido amniótico 25,5 cm), edema subcutáneo, hidrotórax, ascitis (circunferencia abdominal 36,7 $\mathrm{cm}$ ), arteria umbilical única, sexo femenino, ausencia de flujo diastólico de arteria umbilical, vasodilatación compensatoria de la arteria cerebral media. (Figuras 1 a 4). La evaluación del estado fetal fue complementada con prueba no estresante, informado como activo reactivo.

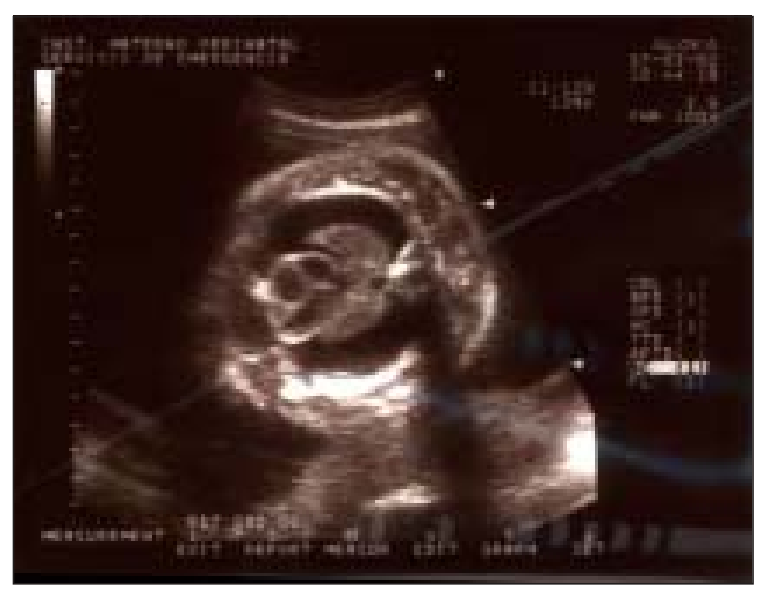

Figura 1. Muestra el gran hidrotórax con marcado compromiso pulmonar

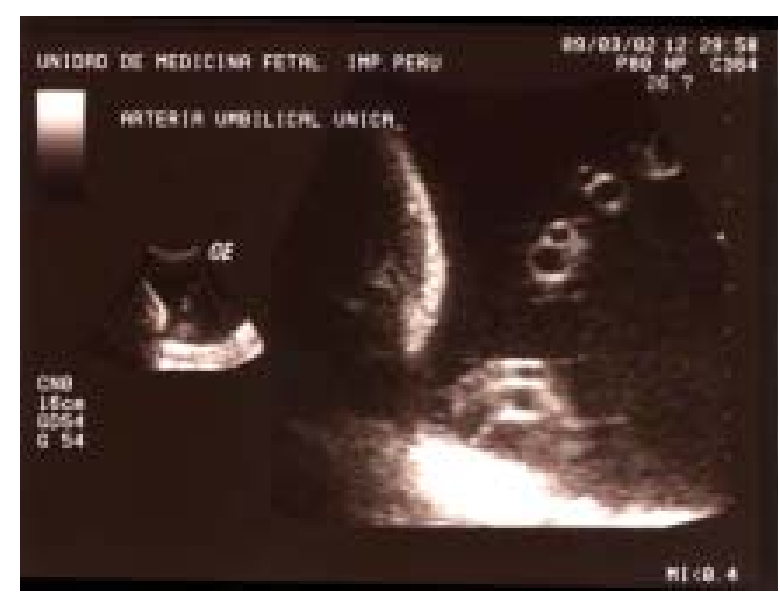

Figura 2. Presencia de arteria umbilical única con polihidramnios
Durante su hospitalización, se determinó el grupo sanguíneo $\mathrm{O}$ y factor $\mathrm{Rh}$ positivo, prueba de Coombs indirecta negativa. La paciente fue dada de alta con feto vivo al $4^{\circ}$ día.

El equipo médico, ante la ausencia de anomalías estructurales mayores, decidió realizar una cordocentesis ambulatoria a los 2 días, para precisar el diagnostico, previa firma del consentimiento informado. Posteriormente, se realizó amniocentesis para aliviar la sintomatología de la paciente, extrayéndose 850 $\mathrm{mL}$ de líquido.

La paciente reingresó al $6^{\circ}$ día del procedimiento por ausencia de movimientos fetales, diagnosticándosele óbito fetal. El parto se produjo vía vaginal de manera electiva al día siguiente, con los hallazgos anatomopatológicos de feto macerado, talla $48 \mathrm{~cm}$, perímetro cefálico 27,2 , perímetro torácico $28,5 \mathrm{~cm}$, perímetro abdominal $31 \mathrm{~cm}$, pie $6 \mathrm{~cm}$, peso 2460 gramos, primer dedo separado del pie, implantación baja de oreja, boca ampliamente abierta con macroglosia, nariz corta, hipertelorismo, piel redundante en la nuca, hipoplasia pulmonar bilateral, severa anasarca; hallazgos compatibles con síndrome de Down (Figuras 5 y 6). E1 cariotipo (Figura 7) de sangre obtenida por cordocentesis, mediante técnica de bandeo

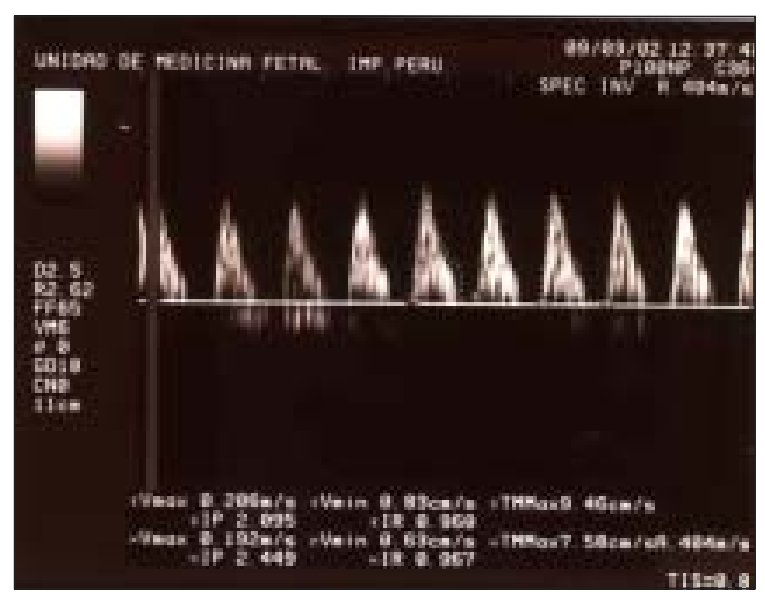

Figura 3. Flujometria Doppler que muestra ausencia de flujo diastólico en arteria umbilical 


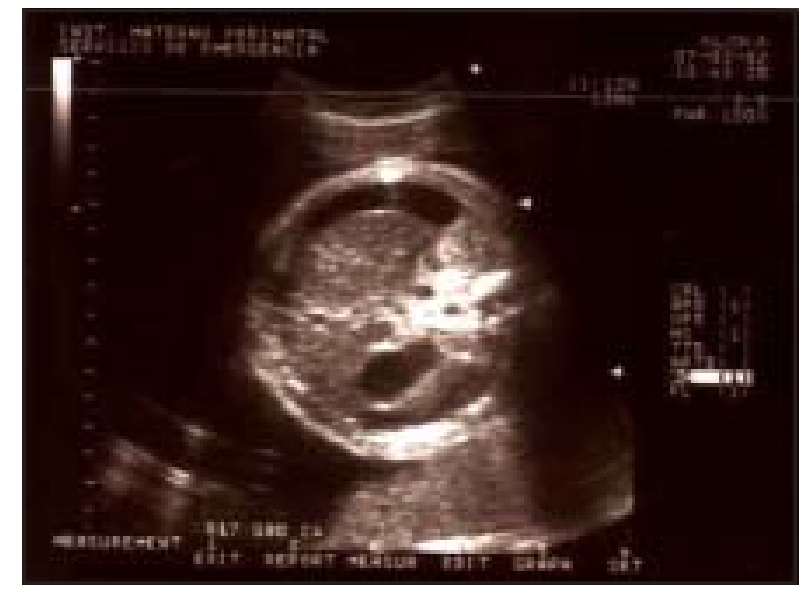

Figura 4. Marcada ascitis en un corte abdominal.

cromosómico GTG, confirmó una trisomía del par 21. La paciente fue dada de alta 2 días más tarde, previa consejería genética.

\section{DISCUSIÓN}

El hidrops fetalis es uno de los 24 marcadores ecográficos de aneuploidía (27). Puede ser

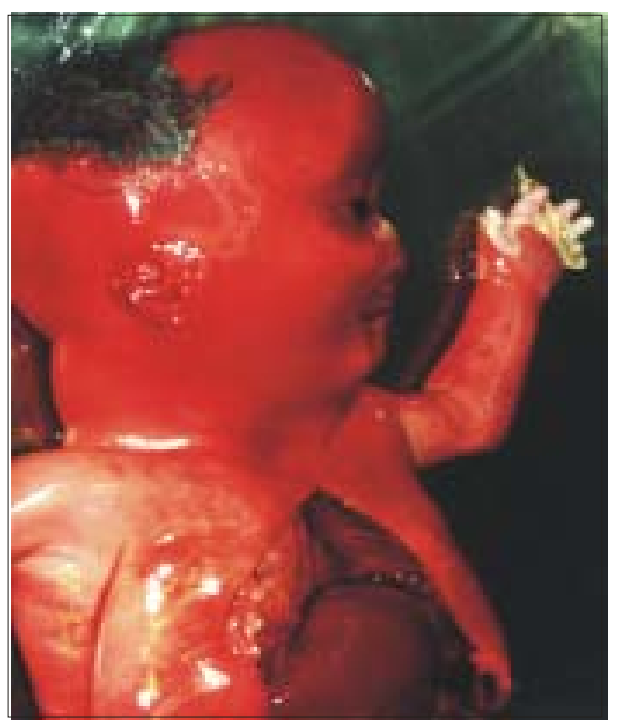

Figura 6. Implantación baja de oreja, piel redundante en nuca, nariz corta y macroglosia.

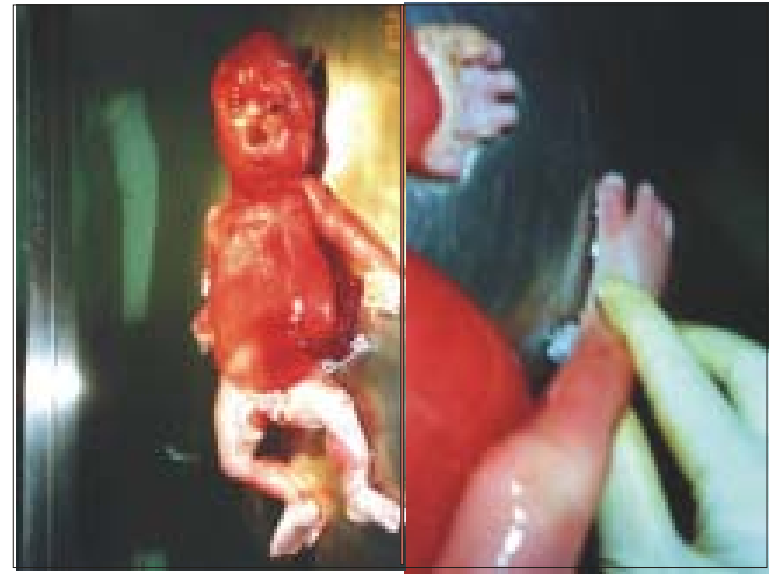

Figura 5. Hidrops fetalis (derecha). Marcada separación del primer dedo (izquierda).

clasificado de acuerdo a su origen en inmune y no inmune. Ecográficamente, ambos tipos son caracterizados por extensa acumulación de fluidos en tejidos fetales o cavidades del cuerpo, reflejando una alteración masiva del balance de agua fetal $\left({ }^{3}\right)$.

En 1943, Potter describió una entidad clínica que afectaba a embarazos sin sensibilización

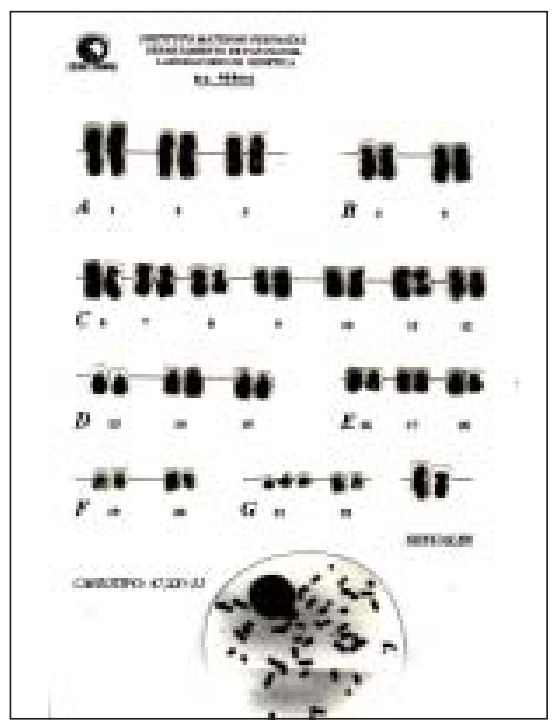

Figura 7. Cariotipo obtenido de la cordocentesis. 
Rh(D) y se caracterizaba por anasarca fetal, edema placentario y, a menudo, derrames serosos en el feto. Potter reconoció que esta entidad, denominada desde entonces hidrops fetalis no inmune, no representaba una enfermedad específica, sino mas bien una manifestación tardía de muchas enfermedades severas. Cuando Potter describió por primera vez el hidrops fetalis no inmune, esta entidad constituía menos del $20 \%$ de los casos de hidrops; en cambio, desde el advenimiento de profilaxis efectiva contra la sensibilizacion $\mathrm{Rh}(\mathrm{D})$, la frecuencia relativa del hidrops fetalis no inmune ha aumentado al $90 \%\left({ }^{4}\right)$.

La incidencia global del hidrops fetal, según Santolaya $\left({ }^{5}\right)$, es de $13 \%$ para la forma inmune y $87 \%$ para la no inmune. La incidencia ecográfica de hidrops fetalis en centros de referencia puede ser tan alto como 1 en 165 embarazos $\left({ }^{5}\right)$. Esta entidad puede ocurrir en poblaciones de riesgo bajo y se la puede detectar con estudio ecográfico a inicios del $2^{\circ}$ trimestre. Mas aun, la complejidad de esta condición y la alta tasa de anormalidades cromosómicas requieren la transferencia a centros de alto riesgo para su evaluación y manejo.

Resultó fácil orientar el diagnóstico no inmune al ser la paciente grupo $\mathrm{O}$ Rh positivo $\mathrm{y}$ con prueba de Coombs indirecta negativa. Sin embargo, nos quedaba una amplia gama de posibilidades causales, según Holzgreve y col. $\left.{ }^{6}\right)$, cardiovasculares, cromosómicas, síndromes malformativos, hematológicas, respiratoria, urinaria, gastrointestinales, hepática, materna, medicamentosa, infecciosa. Otra clasificación etiológica la hace Santolaya $\left(^{5}\right)$ en inmune, intrínseca y extrínseca. Los hallazgos ecográficos y cardiotocográficos alejaron la posibilidad de una cardiopatía, la cual se presenta con mayor frecuencia $(40 \%)\left({ }^{7}\right)$. Debido a que la siguiente causa lo representan las anormalidades cromosómicas (15\%), se procedió a realizar una cordocentesis, a fin de obtener una muestra para estudio citogenético $\left({ }^{8,9}\right)$. En el estudio de las metafases analizadas mediante técnica de bandeo cromosómico GTG, se encontró 47 cromosomas, hallándose un cromosoma 21 adicional. Dado que para esta entidad se cuenta con marcadores ecográficos tales como edema nucal, ausencia del hueso nasal, braquicefalia, aplanamiento de la cara, intestino hiperecogénico, foco hiperecogénico intracardiaco, acortamiento de huesos largos, ectasia piélica, signo de la sandalia, clinodactilia $\left.{ }^{(10-14}\right)$, se debe hacer énfasis en exploraciones ecográficas en el $2^{\circ}$ trimestre, a fin de arribar a un diagnóstico más temprano. Hay que hacer notar que de los marcadores ecográficos antes mencionados, no pudieron detectar el signo de la sandalia y la nariz corta ( ausencia del hueso nasal), probablemente por el marcado edema subcutáneo.

El tratamiento depende de la causa del hidrops. Así, si se debiera a un problema de isoinmunización, se maneja con transfusiones intrauterinas; pero, en casos de hidrops no inmune, el tratamiento puede variar desde una conducta expectante hasta el manejo invasivo (derivaciones, transfusiones). Debido a que la mayoría de lesiones asociadas con estos síndromes son en última instancia fatales para el feto o recién nacido, el conocimiento de la causa es importante para planificar la vía del parto. Para el presente caso, se manejó de manera expectante, con vigilancia estrecha anteparto, con el fin de lograr una mayor madurez, situación con pocas probabilidades por el severo hidrotórax e hipoplasia pulmonar. El tratamiento prenatal de los signos de hidrops mediante paracentesis abdominal fetal y/o punción torácica, sólo tiene sentido de efectividad teórica cuando se va a realizar de inmediato un parto electivo y se requiere descomprimir el abdomen para permitir el parto vaginal, o cuando se observa que el hidrotórax no facilitará la suficiente expansión pulmonar $(1,15,16)$.

Se ha publicado varias comunicaciones sobre transfusión intrauterina para el tratamiento. Así, Sahakian y col, en 1990; informaron un resultado exitoso después de la transfusión intravascular en un feto de 20 semanas con infección por parvovirus B-19 $\left({ }^{15,17}\right)$. En nuestro caso, no se 
pudo evaluar el grado de anemia fetal, por carecer del instrumento apropiado para ello. Sin embargo, se describe un procedimiento no invasivo para determinar la anemia con flujometría Doppler de la arteria cerebral media $\left({ }^{18,19}\right)$.

La tasa de mortalidad del hidrops fetalis no inmune varía entre 50 y $98 \%$, la misma que depende de la causa del problema $\left({ }^{20-25}\right)$. La muerte fetal fue comprobada al $6^{\circ}$ día del procedimiento. Es importante el consejo genético, ya que existen trastornos recesivos autosómicos específicos que ocasionarían hasta un $25 \%$ de recurrencia.

No se produjo complicaciones maternas, como preeclampsia, parto pretérmino, hemorragia posparto, como se describe en algunas series $\left({ }^{26}\right)$.

El presente caso sirve para evaluar la capacidad de diagnóstico antenatal y manejo de patologías fetales como el hidrops en la unidad de Medicina Fetal del IMP y, a la vez, motivar la realización de estudios descriptivos y analíticos sobre el tema.

\section{REFERENCIAS BIBLIOGRÁFICAS}

1. Acien P. Tratado de Obstetricia y Ginecología. $1^{\text {a }}$ ed. Ed Alicante: Ed. Molloy; 1998.

2. IM SS, Rizos N, Joutsi P, et al. Non immunologic hydrops fetalis. Am J Obstet Gynecol 1984;148:566.

3. Saltzman DH, Frigoletto FD, Harloow BL, et al. Sonographic evaluation of hydrops fetalis. Obstet Gynecol 1989;74:106.

4. Warsof SL, Nicolaides KH, Roedeck C. Immune and non immune hydrops. Clin Obstet Gynecol 1986;29:533.

5. Santolaya J, Alley D, Jaffe R, et al. Antenatal classification of hydrops fetalis. Obstet Gynecol 1992;79:256.

6. Holzgreve W, Curry Jr. Non immune hydrops fetalis: Diagnosis and management. Semin Perinatol 1985;9:52.

7. Callen PW. Ecografía en Obstetricia y Ginecología. $3^{\text {a }}$ ed. Ed Panamericana; 1995.

8. Holzgreve W, Curry CJR, Golbus MS, et al. Investigation of non immune hydrops fetalis. Am J Obstet Gynecol 1984;150:805.

9. Hseih FJ, Chang FM, Ko TM, Chen HU. Percutaneuos ultrasound-guided fetal blood sampling in management of non immune hydrops fetalis. Am J Obstet Gynecol 1987; 157:44.
10. Jenderny J. Increased nuchal translucency, hydrops fetalis or hygroma colli. A new test strategy for early fetal aneuploidy detection. Fetal Diagn Ther 2001;16(4):211-4.

11. Smith-Bindman R. Second-trimester ultrasound to detect fetuses with Down syndrome: a meta-analysis . JAMA 2001;285(8):1044-55 .

12. Nyberg DA. Isolated sonographic markers for detection of fetal Down syndrome in the second trimester of pregnancy. J Ultrasound Med 2001;20(10):1053-63 .

13. Vautier-Rit S. Echographic signs of trisomy 21 in the second trimester of pregnancy: actual value after analysis of the literature]. J Gynecol Obstet Biol Reprod (Paris) 2000;29(5):445-53.

14. Simona C, Curcio P, Papageorghiou A, Sonek J, Nicolaides $\mathrm{K}$. Absence of nasal bone in fetuses with trisomy 21 at 1114 weeks of gestation : an observational study. Lancet 2001;358:1665-7.

15. Sahakian V, Weiner CP, Naides SJ, Williamsom RA, Sharosch LL. Intrauterine transfusion treatment of non immune hydrops fetalis secondary to human parvovirus b19 infection. Am J Obstet Gynecol 1991;164:1090.

16. Jones DC. Non immune fetal hydrops. Diagnosis and obstetrical management. Semin Perinatol 1995;19:447-61.

17. Nicolaides K, Soothill PW, Rodeok CH, Clewell W. Rh disease. Intravascular fetal blood transfusion by cordocentesis. Fetal Therapy 1986;1:185-92.

18. Sherif A, Abdel-Fattah, Soothill PW, Carroll SG, Kyle PM. Noninvasive diagnosis of anemia in hydrops fetalis with the use of middle cerebral artery Doppler velocity .Am J Obstet Gynecol 2001;185:1411-5.

19. Teixeira JMA, Duncan K, Letsky E, Fisk NM. Middle cerebral artery peak systolic velocity in the prediction of fetal anemia. Ultrasound Obstet Gynecol 2000;15:205-8.

20. Etches PC, Lemmos JA. Non immune hydrops fetalis: Report of 22 cases including three siblings. Pediatrics 1979;64:326.

21. Hutchison A, Drew JA, Yu V, Williams HL, Fortune DW, Beischer NA. Non immunologic hydrops fetalis: A review of 61 cases. Obstet Gynecol 1982;59:247.

22. Castillo RA, De Vope LD, Hadi HA, Martin S, Geist D. Non immunologic hydrops fetalis. Clinical experience and factors related to poor outcome. Am J Obstet Gynecol 1986;155:812.

23. Mahony BS, Filly RA, Callen PW, Chinn DH, Golbus MS. Severe non immunologic hydrops fetalis: Sonographic evaluation. Radiology 1984;151:757.

24. Watson J, Campbell S. Antenatal evaluation and management in non immune hydrops fetalis. Obstet Gynecol 1986;67:589.

25. Graves GR, Baskett TF. Non immune hydrops fetalis. Antenatal diagnosis and management. Am J Obstet Gynecol 1984;148:563.

26. Bianchi DW. Fetology. Diagnosis and Management of Fetal Patient. Edit. Mac Graw Hill; 2000.

27. Snijders RJM, Nikolaides KH. Ultrasound markers for fetal chromosomal defects. Ed. Parthenon Publishers; 1996. 